\title{
État hydrique et mouvements d'eau dans les tubercules d'Helianthus tuberosus L lors de la dormance et de sa levée
}

\author{
R Sueldo 1, 2, M Gendraud 2, A Coudret 2 \\ 1 Univ nac de Mar Del Plata, ERA-INTA (Balcarce, unidad integrada facultad de ciencias agrarias, \\ CC 276 (7620) Balcarce, Argentina; \\ 2 Université Clermont II, URA CNRS n० 45, laboratoire de phytomorphogenèse, 4 rue Ledru, 63038 Clermont-Ferrand, France
}

(Reçu le 4 janvier 1990; accepté le 9 décembre 1990)

\begin{abstract}
Résumé - L'étude du métabolisme hydrique des tubercules de tompinambour (Helianthus tuberosus $L$ ) dormants, placés soit à $24{ }^{\circ} \mathrm{C}$ (condition de boulage), soit à $4{ }^{\circ} \mathrm{C}$ (condition de levée de dormance) a permis de mettre en évidence le rôle de l'eau dans la dormance et sa levée.

Chez les tubercules maintenus à $24^{\circ} \mathrm{C}$, les modifications de la teneur en eau dans les tronçons 2 et 3 , au $15^{\mathrm{e}} \mathrm{j}$, pourraient être liées à l'initiation du processus de boulage $\left(30^{\mathrm{e}} \mathrm{j}\right)$. L'augmentation du potentiel osmotique et de la teneur en eau, dans la base des tubercules, permet d'envisager que cette base rend possible l'alimentation en sucres et probablement en eau lors de la croissance tubérisée.

Chez les tubercules maintenus à $4{ }^{\circ} \mathrm{C}$, après $9-10$ semaines de séjour au froid, d'importantes modifications ont lieu : il y a un afflux d'eau et de matières de réserve vers le bourgeon.
\end{abstract}

paramètre hydrique / eau / levée de dormance / tubercule / Helianthus tuberosus = topinambour / conservation

Summary - Water status and water movements in tubers of Helianthus tuberosus L during dormancy and breaking of dormancy. The study of water parameters in dormant Jerusalem artichokes tubers (Helianthus tuberosus L) kept at $24^{\circ} \mathrm{C}$ ("boulage" process) and at $4{ }^{\circ} \mathrm{C}$ (to break the dormancy), in darkness showed the role of water during dormancy and the breaking period. During the first $15 \mathrm{~d}$ at $24{ }^{\circ} \mathrm{C}$, the water content in the region adjacent to the bud increased (fig 4a), and that in the middle region decreased (fig 3a); this could indicate water movement. The fact that osmotic potential in the basal region increased (fig 2b) and that its water potential was higher than in the middle region (fig 2c) could indicate that the basal region supplied the middle region with sugars and probably with water. The water content increase in the basal region (fig 2a) could explain the increase of osmotic potential in this same region. In tubers kept at breaking conditions of dormancy at $4{ }^{\circ} \mathrm{C}$, during the first weeks the same variations in basal and middle regions were observed. The differences between both treatments $\left(24{ }^{\circ} \mathrm{C}\right.$ and $\left.4{ }^{\circ} \mathrm{C}\right)$ were found to be the variation range and the speed at which the variations took place. After these first wk at $4{ }^{\circ} \mathrm{C}$, the decrease in the osmotic potential of the bud (fig $7 b$ ) led to an increase in bud water potential (fig $7 c$ ); this could indicate water supply by the bud to the adjacent region and explain the increase in turgor pressure of the bud and the water content (fig 7a).

It was therefore concluded that after 9-10 wk at $4{ }^{\circ} \mathrm{C}$, significant changes occurred in water status of Helianthus tuberosus $L$ tubers.

water parameter / breaking of dormancy / tuber $/$ Helianthus tuberosus = Jerusalem artichoke $/$ conservation

\section{INTRODUCTION}

Bon nombre de plantes supérieures se multiplient par l'intermédiaire d'organes végétatifs plus ou moins spécialisés; ces organes de conservation et de multiplication sont toujours constitués par un ensemble de bourgeons en liaison directe avec des réserves souvent glucidiques. Dans le topinambour, le stockage se traduit par l'hypertrophie radiale de parenchymes d'origine primaire : il y a tubérisation (Courduroux, 1967). Développé au cours du printemps et de l'été, le tubercule est, d'octobre à février, dormant; cette dormance est progressivement affaiblie par le froid; la température joue donc un rôle important dans l'installation et le maintien de la dormance.

Des tubercules dormants de topinambours, placés à l'obscurité, à $24^{\circ} \mathrm{C}$, présentent un phénomène de boulage qui est une croissance tubérisée d'un germe, en conséquence d'une levée 
partielle de dormance. La dormance peut être levée par un séjour de 16 semaines à $4{ }^{\circ} \mathrm{C}$, à l'obscurité, ce qui se traduit par le développement d'une tige étiolée à partir du bourgeon apical (Gendraud, 1977).

On considère actuellement que les parenchymes dormants absorbent et stockent les nutriments au détriment de l'alimentation du bourgeon, ce qui créerait des carences dans les zones méristématiques et donc, empêcherait leur bon fonctionnement; les parenchymes des tubercules dormants ont des potentialités plus importantes que ceux des non dormants, via une activité électrogène plus forte au niveau du plasmalemme de la cellule parenchymateuse et de la présence très probable d'associations plasmalemme-tonoplaste (Petel, 1986).

II doit y avoir, par ailleurs, des interactions inhibitrices entre les territoires en croissance et le parenchyme sous-jacent, entre les compartiments intracellulaires et entre différentes régions du tubercule, par l'intermédiaire d'hormones (Tort et al, 1985).

Les flux de nutriments, d'hormones, doivent être concomitants avec des mouvements d'eau à l'intérieur des tubercules, puisqu'en dessous de certaines valeurs de teneurs en eau relatives, la croissance est inhibée (Sinclair et Ludlow, 1985) : l'expression ou l'inhibition d'un signal (débourrement ou non débourrement d'un bourgeon, par exemple) est corrélée à ce paramètre (Coudret et al, 1987).

Les échanges d'eau s'effectuent selon des gradients de potentiels hydriques dépendants de la pression osmotique des cellules et donc des mécanismes d'absorption et des structures membranaires intervenant dans la régulation du potentiel de turgescence. Un abaissement consécutif du potentiel de turgescence et du potentiel hydrique des cellules entraîne la formation d'un gradient de potentiel hydrique favorable à un flux d'entrée d'eau dans les cellules en croissance (Lockart, 1965). Dans le même temps, l'extension cellulaire n'est possible que si le potentiel de turgescence dépasse un certain seuil en dessous duquel les processus métaboliques nécessaires au relâchement de la paroi sont inhibés (Cosgrove et Cleland, 1983). L'extension cellulaire induit en même temps un gradient de potentiel hydrique entre le xylème et la cellule, qui empêche le potentiel de turgescence d'atteindre son maximum; ceci permet l'entrée d'eau dans les cellules à une vitesse compatible avec la croissance cellulaire (Katou et Furumoto, 1986; Nonami et Boyer, 1987).
La division cellulaire, le grandissement cellulaire et l'accumulation des sucres dans les régions en croissance doivent impliquer un système de transport efficace des précurseurs glucidiques. Ce transport pourrait être lié au transport ou au mouvement de l'eau dans le tubercule. Un tel mouvement d'eau et de sucres doit provoquer des modifications dans la plupart des paramètres hydriques, tout au long du tubercule, pendant le boulage ou la levée de dormance.

C'est pour celà que ce travail étudie le métabolisme hydrique dans les différentes parties du tubercule, sur des tubercules placés en conditions permettant, soit la croissance longue, soit la croissance tubérisée, et ce pendant toute la durée du séjour des tubercules dans ces conditions.

Quels sont les flux d'eau se développant dans les tubercules pendant le séjour au froid (conditions de levée de dormance) ? Comment ces flux d'eau sont-ils liés à l'expression soit d'une tubérisation, soit d'une pousse longue?

\section{MATÉRIEL ET MÉTHODES}

Les tubercules sont multipliés dans le jardin du laboratoire. Plantés à la fin du mois de mars à l'état non dormant dans une terre aussi légère que possible, ils germent immédiatement et, à la fin du mois de juin, les jeunes plants émettent leurs premiers stolons souterrains dont l'extrémité se renfle dès la fin du mois de juillet. L'édification des nouveaux tubercules se poursuit pendant les mois d'août et septembre. La plantation est irriguée pendant les mois de juillet, août et septembre en cas de forte chaleur sèche. Après suppression des parties aériennes, les nouveaux tubercules dormants sont récoltés à partir du mois de novembre et placés dans du sable maintenu humide $(\approx 5 \%$ d'eau par rapport au poids de matière sèche, en chambre climatisée à $24^{\circ} \mathrm{C}$ ou $4^{\circ} \mathrm{C}$ ).

Les paramètres hydriques étudiés : teneur en eau, potentiel osmotique, potentiel hydrique et potentiel de turgescence sont analysés toutes les 2 semaines pendant 4 mois. La distribution des tronçons qui ont été prélevés chaque quinzaine, tout au long du tubercule est présentée dans la figure 1. Le tronçon 1 est le bourgeon, le tronçon 2, la partie qui est juste en dessous du bourgeon, les tronçons 3 et 4 correspondant au milieu du tubercule et les tronçons 5 et 6 à sa base. Un cylindre central du tubercule est prélevé à l'aide d'un emporte-pièce et ce cylindre est ensuite rapidement découpé en tronçons. Les méthodes d'étude des paramètres hydriques étant destructives, c'est sur des lots différents, mais comparables, que ces paramètres sont étudiés. 


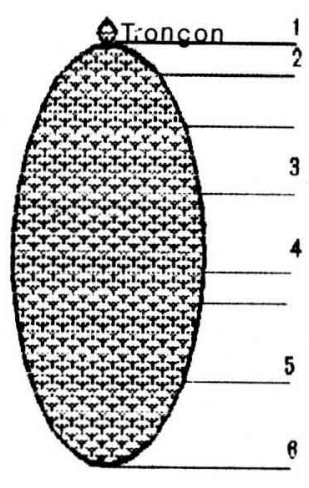

Fig 1. Représentation schématique d'un tubercule de topinambour indiquant la position des tronçons étudiés.

\section{Teneur en eau}

C'est la différence entre le poids de matière fraîche et celui de la matière sèche obtenue par dessication à $80^{\circ} \mathrm{C}$ jusqu'à un poids constant $(24 \mathrm{~h})$; cette différence est rapportée à 100 unités de matière sèche. Elle est donnée par la relation suivante : $100(P F-P S) / P S$. Les valeurs obtenues sont la moyenne de 6 mesures.

\section{Potentiel hydrique $(=\psi)$}

C'est un paramètre qui permet de caractériser l'état hydrique d'un végétal (Eckart et al, 1977) dans lequel il règle les mouvements de l'eau; il est égal mais de signe opposé à l'énergie qu'il faut appliquer aux tissus d'une plante pour en libérer une mole d'eau. II est mesuré à l'aide d'un psychromètre type Wescor : le tronçon est prélevé et enfermé dans une chambre Wescor en $10 \mathrm{~s}$. Les valeurs obtenues sont des moyennes portant sur 3 échantillons différents.

\section{Potentiel osmotique $(=\pi)$}

Il caractérise le degré d'imbibition du protoplasme (Kramer, 1969) : il dépend des substances solubles réparties dans les vacuoles, mais aussi de celles qui sont présentes dans le cytoplasme. Pour son évaluation, on admet que dans une cellule en équilibre, le potentiel matriciel du cytoplasme est égal au potentiel osmotique de la vacuole, le tonoplaste étant supposé hémiperméable (Coudret, 1979). Le potentiel osmotique est mesuré à l'aide d'un cryoscope électronique après traitement par l'eau bouillante des morceaux de tubercules et filtration. II est estimé en fonction de la différence entre la température de congélation du suc cellulaire et celle de l'eau pure. Au cours de l'extraction, l'hydrolyse de molécules non osmotiquement actives peut produire des substances actives osmotiquement (glucose, fructose), recueillies dans le filtrat et responsables d'une surestimation du potentiel osmoti- que (Houchi, 1986). Les valeurs obtenues sont des moyennes portant sur 3 échantillons différents.

\section{Potentiel de turgescence (= P)}

La paroi pectocellulosique forme un cadre plus ou moins rigide qui exerce une pression, dite de turgescence, sur le contenu cellulaire. La différence de pression entre la cellule et la source potentielle en eau détermine la possibilité pour l'eau d'y entrer. $P$ est calculé en fonction des 2 paramètres précédents à partir de la relation $\psi=\pi+P$.

Pour chaque résultat, il a été estimé l'intervalle de confiance pour un degré de sécurité de $95 \%$.

\section{RÉSULTATS}

\section{Tubercules maintenus à $24^{\circ} \mathrm{C}$}

La teneur en eau augmente significativement (fig 2a) dans la base des tubercules (tronçons 5 et $6: 89 \%$ et $177 \%$ ), ce qui peut expliquer l'augmentation observée du potentiel osmotique (fig $2 b)$. Le potentiel hydrique du tronçon 6 est toujours plus élevé que celui du tronçon 5 (fig 2c), ce qui permet de penser que de l'eau peut circuler vers le tronçon 5 .

Dans la partie centrale des tubercules (tronçons 3 et 4 ), la teneur en eau reste stable dans le tronçon 4 pendant $30 \mathrm{j}$ (fig $3 \mathrm{a}$ ), puis augmente jusqu'au $60^{e} \mathrm{j}(134 \%$ ); dans le tronçon 3 , elle diminue pendant les 15 premiers j $(26 \%)$ et reste stable jusqu'au $45^{\mathrm{e}} \mathrm{j}$ : de l'eau circule soit du tronçon 3 vers le tronçon 4 , soit du tronçon 3 vers la partie apicale des tubercules; à partir du $45^{\mathrm{e}} \mathrm{j}$, la teneur en eau du tronçon 3 augmente (88\%). Dans le même temps, les potentiels osmotiques restent stables (fig $3 b$ ). La plupart du temps, les valeurs du potentiel hydrique des tronçons 3 et 4 sont plus faibles que celles des tronçons 2, 5 et 6 (fig 3c); ceci paraît indiquer que de l'eau circule vers les tronçons 3 et 4 . Au $15^{\mathrm{e}} \mathrm{j}$, les différences sont significatives, mais les résultats obtenus pour tous les tronçons montrent qu'il y a toujours un gradient de potentiel hydrique du tronçon 2 vers le milieu du tubercule ainsi que de la base vers la partie médiane. Au fur et à mesure que le boulage se développe, les cellules de la base et de la partie médiane se plasmolysent (elles paraissent perdre de l'eau et des sucres). Le potentiel de turgescence reste proche de 0 .

Dans la partie subterminale des tubercules (tronçon 2), la teneur en eau augmente (fig 4a) 

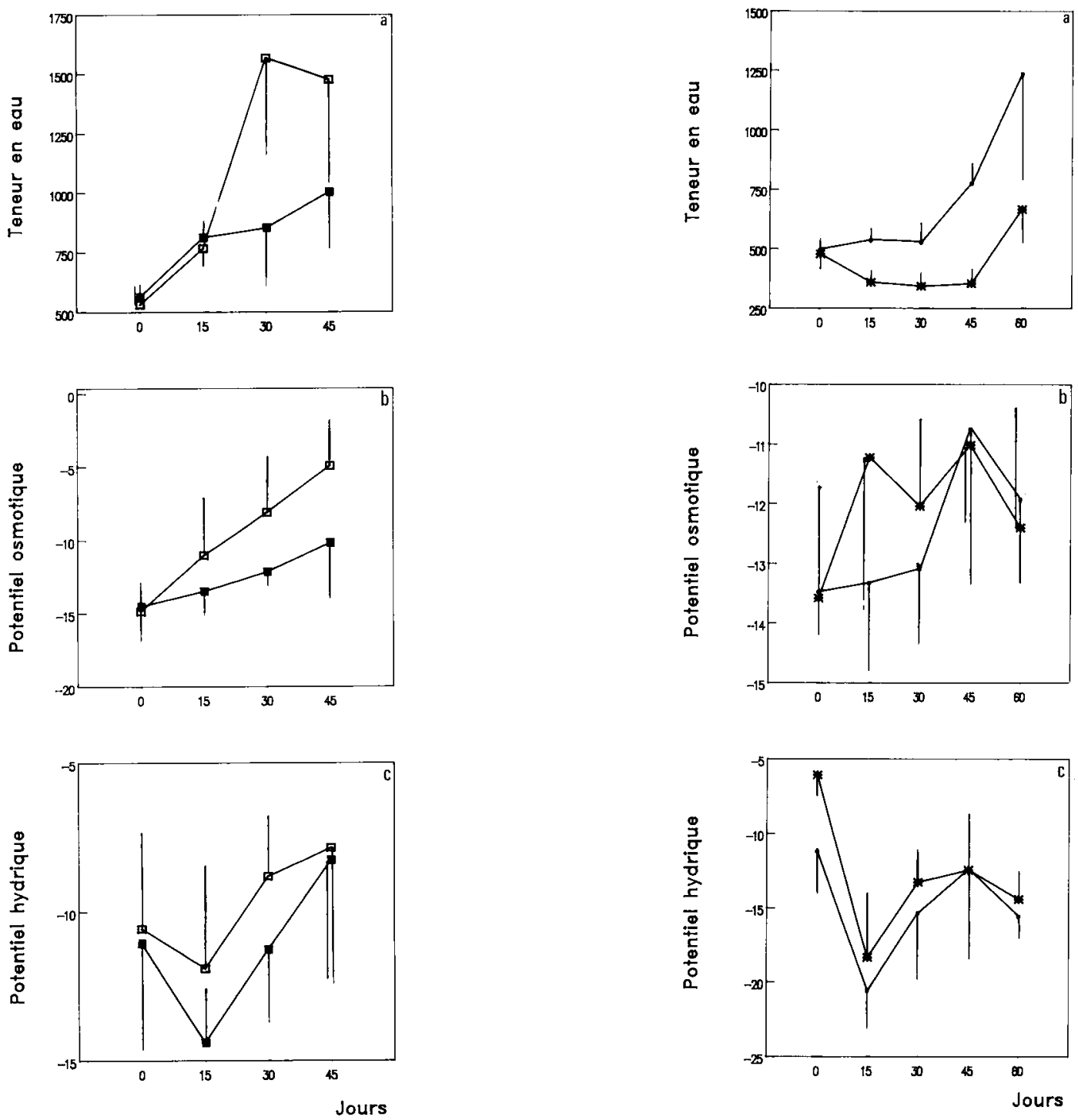

Fig 2. a Variations de la teneur en eau (quantité d'eau contenue par rapport au poids de matière sèche, en \%) et des potentiels $\mathbf{b}$ osmotique et $\mathbf{c}$ hydrique (exprimés en bar), en fonction du temps (en j) dans les tronçons 5 ( $\square$ ) et $6(\square)$, chez le topinambour à $24^{\circ} \mathrm{C}$.

pendant $15 \mathrm{j}(65 \%)$. Ceci pourrait indiquer que de l'eau y arrive : - soit à partir du tronçon 3 (fig 3a); - soit à partir du sable. Ensuite, elle diminue jusqu'au $45 \mathrm{e}$ j et augmente au-delà (140\%). Le potentiel osmotique ne varie pas significativement (fig 4b); le potentiel hydrique reste stable pendant $45 \mathrm{j}$; il diminue au $60 \mathrm{e}$ j (fig 4c). II n'y a pas de différence significative en ce qui concerne le potentiel de turgescence.

Les résultats concernant le bourgeon (tronçon 1) ne sont pas discutés car celui-ci, à partir du $30 \mathrm{e}$ j permet la formation du tubercule fils.

Lors du boulage, dans les tubercules fils, les variations des paramètres hydriques indiquent une arrivée de sucres solubles et probablement

d'eau venant du tubercule mère (résultats non publiés).

\section{Tubercules maintenus à $4{ }^{\circ} \mathrm{C}$}

Dans la base des tubercules (tronçons 5 et 6 ), la teneur en eau augmente jusqu'au $60^{\mathrm{e}} \mathrm{j}$ (fig $5 \mathrm{a}$ ); au $60 \mathrm{e} \mathrm{j}$, le potentiel osmotique augmente (fig $5 b)$. Le potentiel de turgescence ne varie pas et reste voisin de 0 . Le potentiel hydrique des 2 tronçons diminue significativement au $45^{\mathrm{e}}$ et au $60 \mathrm{e}$ j; ultérieurement, il augmente significativement (fig 5c).

Dans la partie médiane des tubercules (tronçons 3 et 4 ), la teneur en eau (fig 6a) et le poten- 

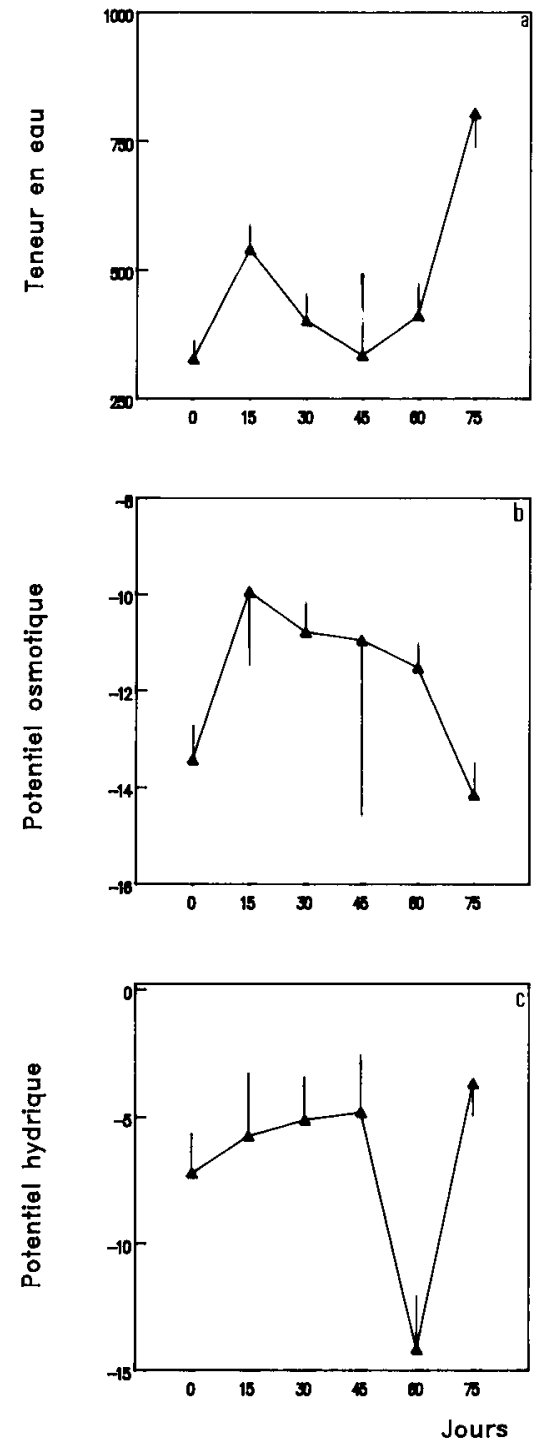

Fig 4. a Variations de la teneur en eau, des potentiels b smotique et $c$ hydrique, en fonction du temps (en j) dans le tronçon 2 chez le topinambour à $24^{\circ} \mathrm{C}$.

tiel osmotique (fig 6b) ne varient pas. II est à noter qu'après $105 \mathrm{j}$ de séjour à $4{ }^{\circ} \mathrm{C}$, le potentiel hydrique du tronçon 3 (fig $6 \mathrm{c}$ ) est plus élevé que celui du tronçon 2 (fig $7 \mathrm{c}$ ). Le potentiel de turgescence ne varie pas et reste toujours voisin de 0 .

Dans la partie subterminale, la teneur en eau (fig 7a) augmente significativement pendant les premiers 60 jours, puis a une tendance à diminuer. Le potentiel osmotique (fig $7 \mathrm{~b}$ ) augmente entre le $15^{\mathrm{e}}$ et le $75^{\mathrm{e}} \mathrm{j}$ et diminue nettement au $90 \mathrm{j}$. Le potentiel hydrique (fig 7c) diminue audelà de $45 \mathrm{j}$, et au $105^{\mathrm{e}} \mathrm{j}$ il est plus faible que celui du tronçon 3 : de l'eau peut donc migrer du tronçon 3 vers le tronçon 2 .

Dans le bourgeon, la teneur en eau augmente du $75^{\mathrm{e}}$ au $105^{\mathrm{e}}$ j (fig $7 \mathrm{a}$ ), le potentiel osmotique évolue à l'opposé de la teneur en eau (fig
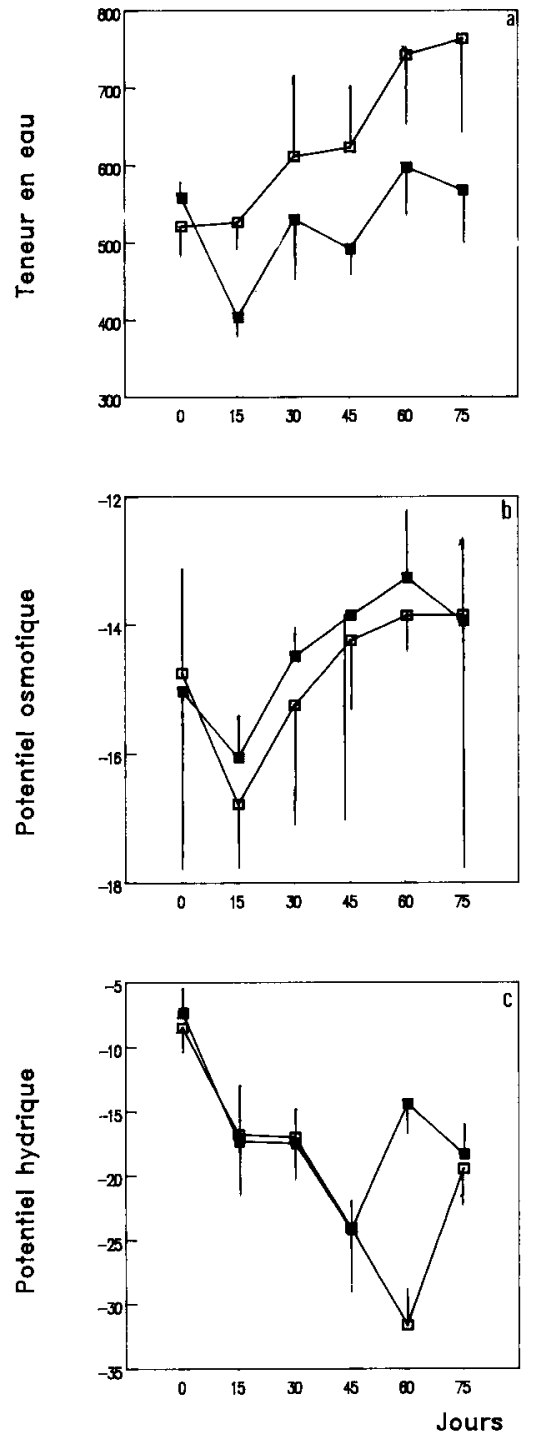

Fig 5. a Variations de la teneur en eau, des potentiels b osmotique et $\mathbf{c}$ hydrique, en fonction du temps (en j) dans les tronçons $5(\square)$ et $6(\square)$ chez le topinambour à $4^{\circ} \mathrm{C}$.

7b). Le potentiel hydrique augmente au $90^{\mathrm{e}}$ jour (fig 7c).

\section{DISCUSSION}

\section{Tubercules dormants placés à $24^{\circ} \mathrm{C}$}

Pendant les 15 premiers j de séjour à $24^{\circ} \mathrm{C}$, il y a une augmentation significative de la teneur en eau dans la région sousapicale (fig 4a) et diminution dans la partie médiane (fig 3a). Le potentiel hydrique de la partie subapicale (fig 4c), plus fort que celui de le partie médiane (fig $3 c$ ), semble interdire une alimentation en eau à partir de cette dernière, à moins qu'il n'y ait intensifica- 

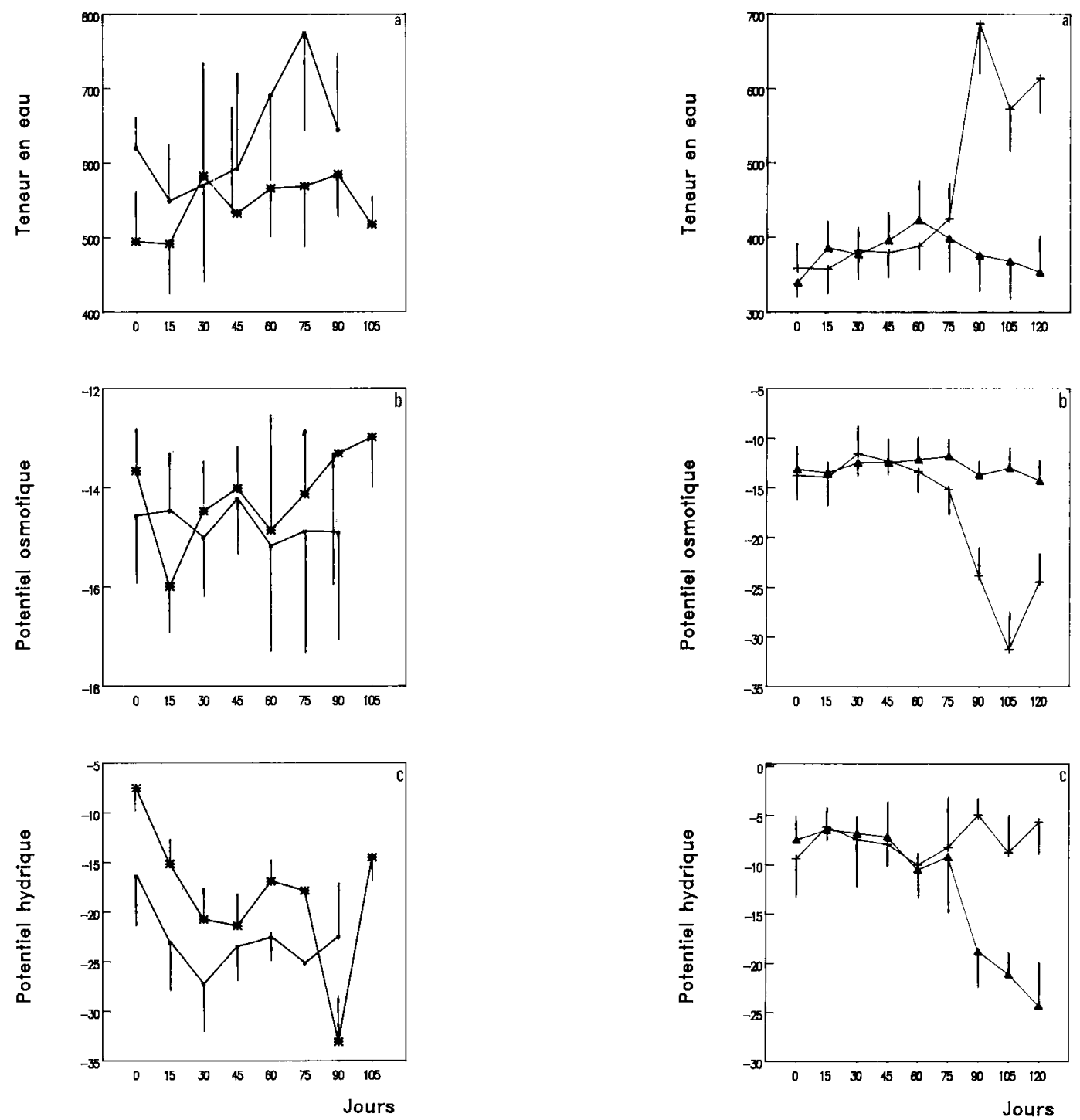

Fig 6. a Variations de la teneur en eau, des potentiels b osmotique et $\mathbf{c}$ hydrique, en fonction du temps (en j) dans les tronçons $3(*)$ et $4\left({ }^{\circ}\right)$ chez le topinambour à $4^{\circ} \mathrm{C}$.

tion de la respiration dans la partie sousapicale, entraînant un gradient hydrique très localisé (Katou et Furumoto, 1986); $15 \mathrm{j}$ après, le boulage commence. La diminution de la teneur en eau observée dans la partie médiane pourrait aussi résulter d'un afflux de sucres solubles provenant de la base du tubercule ou d'une hydrolyse d'inuline dans la partie médiane.

Le fait que la base des tubercules ait un potentiel osmotique qui augmente et que son potentiel hydrique soit toujours plus élevé que celui de la partie médiane pourrait indiquer que cette base participe à l'alimentation du reste du tubercule en sucres et problablement en eau.

L'arrivée de sucres et d'eau nécessaires pour la croissance tubérisée, dans les parties médianes et surtout dans la partie sous-apicale,

pourraît entraîner l'augmentation de la teneur en eau observée, tandis que le potentiel osmotique décroît très faiblement (fig $4 b$ ). En même temps, il y a synthèse d'inuline accompagnant la formation du tubercule fils (Teppaz-Mission, 1975). Mais du fait que l'invertase a une faible activité, pour expliquer ce qui doit permettre le boulage il est possible d'envisager 2 hypothèses :

- des associations plasmalemme-tonoplaste permettant le passage direct du saccharose de l'apoplaste à la vacuole (Kaeser, 1983; Roche, communication personnelle);

- des transporteurs du saccharose et des hexoses présents sur le plasmalemme et le tonoplaste, dont l'activité serait dépendante : - soit de la température; - soit de l'environnement phospholipidique : ceci minimiserait le rôle de 
l'hydrolyse plasmalemienne du saccharose dans l'accumulation ou l'obtention d'énergie. Il est déjà connu que dans les racines immatures de betteraves à sucre, le saccharose et les hexoses sont transportés par des transporteurs spécifiques, via un cotransport avec $\mathrm{H}^{+}$(Lemoine et al, 1988); de plus, chez le topinambour, il a été proposé un cotransport actif $\mathrm{H}^{+} /$sucres (Gendraud et Lafleuriel, 1983). Enfin, Frehner et al (1987) et Darwen et John (1989) ont proposé l'existence d'un transporteur du saccharose et d'un transporteur du fructose sur le tonoplaste du topinambour.

Une hydrolyse (dans la base du tubercule) et une synthèse d'inuline (dans les régions en croissance) peuvent être envisagées sous contrôle des potentiels osmotiques; il a été proposé chez le crosne du Japon (Stachys sieboldi, Mig), pendant le boulage, une dépendance du métabolisme de l'amidon au potentiel osmotique du sac vacuolaire (Vandeven, 1982).

Ici, l'augmentation de la teneur en eau dans les tronçons 5 et 6 (fig 2a) pourrait déterminer l'augmentation du potentiel osmotique (fig 2b), particulièrement dans le tronçon 5 . Cet afflux d'eau déclencherait l'hydrolyse de l'inuline afin :

- de maintenir à un niveau adéquat le potentiel osmotique, ou

- de mobiliser les sucres solubles formés par l'hydrolyse vers l'apex pour y être stockés et/ou dégradés.

Chez le crosne du Japon, pendant le boulage, les régions en croissance induisent un appel important de sucres à partir des régions sousjacentes, ce qui détermine une dégradation de l'amidon accumulé (partie médiane et basale) (Vandeven, 1982). Ceci permettrait d'expliquer la stabilité du potentiel osmotique, dans le tronçon 2 , malgré la très forte augmentation de la teneur en eau lors du $75^{\mathrm{e}} \mathrm{j}$.

Tout semble se passer comme s'il existait un seuil de potentiel osmotique au-delà duquel se produit la synthèse et en deçà duquel se déclenche la dégradation.

\section{Tubercules dormants placés à $4{ }^{\circ} \mathrm{C}$}

Pendant la première partie du séjour au froid, dans la base du tubercule, tout se passe comme dans les tubercules maintenus à $24^{\circ} \mathrm{C}$. Les variations du potentiel osmotique et hydrique sont conformes à cette hypothèse. La différence entre les 2 traitements $\left(24^{\circ} \mathrm{C}\right.$ et $\left.4^{\circ} \mathrm{C}\right)$ réside dans l'ampleur des variations et de la vitesse à la- quelle ces modifications ont lieu. Après $90 \mathrm{j}$ de séjour au froid, les cellules de la base sont plasmolysées.

Les faibles valeurs de potentiel hydrique dans la partie médiane (tronçons 3 et 4) (fig 6c) peuvent indiquer que de l'eau y arrive; cette eau pourrait avoir son origine dans les régions périphériques, basales et apicales. À la fin du séjour au froid, le potentiel hydrique du bourgeon reste stable (fig 7c) tandis que celui du tronçon sousjacent diminue fortement : il peut être envisagé une alimentation en eau du bourgeon à partir du sol, liée à la diminution de son potentiel osmotique (fig $7 b$ ). Cette diminution pourrait être due :

- soit à un afflux de sucres lié au fonctionnement des ATPases, à partir de l'apoplaste des bourgeons vers le cytoplasme des cellules de celui-ci; ce mécanisme serait comparable à celui intervenant pendant le boulage dans les tubercules dormants de topinambour et de crosne du Japon (Tort et al, 1985); de plus, il existe une liaison entre afflux d'eau et respiration (Katou et Furumoto, 1986);

- soit à la dégradation de l'inuline des parois, fournissant des sucres solubles aux bourgeons : on sait que la paroi dans le cas des tubercules, peut être considérée comme un compartiment de stockage (Tort et al, 1985);

- soit à l'apparition de sucres solubles dans les bourgeons à partir d'autres polysaccharides des parois.

Cette forte diminution du potentiel osmotique dans le bourgeon, à la fin du séjour au froid, son potentiel hydrique restant stable, serait aussi responsable de l'augmentation du potentiel de turgescence. En effet, il passe de 6,9 bar au $75^{\mathrm{e}}$ j à 18,9 bas au $90^{\circ} \mathrm{j}$.

Le fait que le bourgeon puise de l'eau dans le sol permettrait aussi d'expliquer la forte augmentation de la teneur en eau (fig 7a).

Finalement, il est intéressant de noter que l'augmentation des potentiels hydriques, qui progressent au cours du temps à partir de la base vers la partie médiane, permet d'envisager un relargage de l'eau précédemment liée, sous forme d'eau plus libre, ce qui rendrait cette dernière disponible pour le fonctionnement métabolique. Ceci est comparable avec ce qui se passe chez le frêne, où le potentiel hydrique du point végétatif s'élève sensiblement avant même que la réhydratation du bourgeon n'ait lieu. Ceci est interprété comme le résultat de l'apparition d'eau libre à partir d'eau précédemment liée; cette eau devient alors disponible pour le métabolisme 
(Cottignies, 1987). Pendant la période de dormance, il doit y avoir, dans le tubercule, des métabolites vacuolaires qui immobilisent de l'eau. La dégradation de ces métabolites aurait pour conséquence de rendre l'eau plus libre et disponible pour la croissance.

\section{CONCLUSION}

Chez les tubercules maintenus à $24^{\circ} \mathrm{C}$, l'alimentation de l'apex en métabolites et en eau se ferait mal, l'essentiel des sucres étant mobilisé pour assurer le fonctionnement de la respiration cellulaire; peu de sucres et peu d'eau arriveraient au bourgeon, ce qui ne lui permettrait qu'une croissance lente, tubérisée.

Chez les tubercules, maintenus à $4^{\circ} \mathrm{C}$, les parois joueraient un rôle de réservoir de sucres (inuline ou autres polysaccharides).

La diminution du potentiel osmotique des bourgeons doit entraîner un flux d'eau à partir du sol qui permet une forte augmentation du potentiel de turgescence : $2-3$ semaines plus tard, les bourgeons pourront se développer. Tout se passe comme si le potentiel de turgescence devait atteindre une valeur seuil permettant de mettre en place les processus métaboliques nécessaires au relâchement de la paroi, ce qui permettra aux cellules d'absorber l'eau nécessaire (Cosgrove et Cleland, 1983).

\section{RÉFÉRENCES}

Cosgrove DJ, Cleland RE (1983) Osmotic properties of Pea internodes in relation to growth and auxin action. Plant Physiol 72, 332-338

Cottignies A (1987) Dormance. Ann Sci Nat Bot Biol Vég (Paris) XIII, T 8, 93-142

Coudret A (1979) Action au chlorure de sodium et des antitranspirants sur les échanges d'eau et de gaz carbonique d'un halophyte (Plantago maritima L var Graminae) et d'un glycophyte (Plantago lanceolata L) Thèse Doct Etat Univ Caen, $168 p$

Coudret A, Desbiez MO, Chanteloupe F (1987) Action de l'humidité relative de l'air sur l'expression de corrélations entre bourgeons cotylédonaires, induite par un signal traumatique chez Bidens pilosus L : relation avec la transpiration. $C R$ Séances Acad Sci (Paris) 305, Série III, 633-637

Courduroux JC (1967) Étude du mécanisme physiologique de la tubérisation chez le Topinambour ( $\mathrm{He}$ lianthus tuberosus L). Ann Sci Nat Bot Biol Vég VIII, 215-356
Darwen C, John P (1989) Localization of the enzymes of fructan metabolism in vacuoles isolated by a mechanical method from tubers of Jerusalem Artichoke (Helianthus tuberosus L). Plant Physiol 89, 658-663

Eckart EF, Berger A, Methy M, Heim G, Sauvezon R (1977) Interception de l'énergie rayonnante, échanges de $\mathrm{CO}_{2}$, régime hydrique et production chez différents types de végétations sous climat méditerranéen. In : Les processus de la production végétale primaire, Gauthier - Villars, 1-75

Frehner M, Keller F, Matile P, Wiemken A (1987) Sugar transport across the tonoplast of vacuoles isolated from protoplasts of Jerusalem Artichoke tubers. In: Plant vacuoles (B Marin, ed) Plenum, New-York, 281-286

Gendraud M (1977) Étude de quelques aspects du métabolisme des nucléotides des pousses de topinambour en relation avec leurs potentialités morphogénétiques. Physiol Vég 15, 121-132

Gendraud M, Lafleuriel J (1983) Caractéristiques de l'absorption du saccharose et du tétraphénylphosphonium par les parenchymes de Topinambour, dormants et non dormants, cultivés in vitro. Physiol Vég 21, 1125-1133

Houchi $R$ (1986) Action de la salinité du milieu ( $\mathrm{NaCl}$ ou $\mathrm{Na}_{2} \mathrm{SO}_{4}$ ) sur les relations hydriques, ioniques et échanges gazeux de Plantago maritima L (Halophyte) et de Plantago lanceolata L (Glycophyte). Thèse Doct Ing, Paris VII, $111 \mathrm{p}$

Kaeser W (1983) Ultrastructure of storage cells in Jerusalem artichoke (Helianthus tuberosus $\mathrm{L}$ ). Vesicle formation during inulin synthesis. Pflanzenphysiologie 111, 253-260

Katou K, Furumoto M (1986) A mechanism of respiration-dependent water uptake enhanced by auxin. Protoplasma 133, 174-185

Kramer PJ (1969) Plant and soil water relationships. Mc Graw Hill Book Company. New York

Lemoine R, Daie J, Wyse R (1988) Evidence for the presence of a sucrose carrier in immature sugar beet tap roots. Plant Physiol 86, 575-580

Lockart JA (1965) An analysis of irreversible plant and cell elongation. $J$ Theor Biol 8, 264-276

Nonami H, Boyer JS (1987) Origin of growth-induced water potential: solute concentration is low in apoplast of enlarging tissues. Plant Physiol 83, 596-601

Petel G (1986) Étude comparée, chez le tubercule dormant et non dormant de Topinambour (Helianthus tuberosus L) de l'ATPase et du système transporteur d'électrons liés sur plasmalemme de la celule parenchymateuse. Thèse doct univ Clermont II, $116 \mathrm{p}$

Sinclair TR, Ludlow MM (1985) Who thaught plants thermodynamics? The unfulfilled potential of plant water potential. Aust J Plant Physiol 12, 213-217

Teppaz-Misson C (1975) Contribution à la recherche de marqueurs biochimiques caractéristiques de la 
tubérisation et de la dormance. Activités invertase et saccharose fructosyl transferase liées à l'édification, l'entrée en dormance et la levée de dormance de tubercules de topinambour (Helianthus tuberosus $\mathrm{L}$ ). Thèse doc État Univ Clermont II, $61 \mathrm{p}$

Tort M, Gendraud M, Courduroux JC (1985) Mechanisms of storage in dormant tubers: correlative as- pects, biochemical and ultrastructural approaches. Physiol Veg 23, 289-299

Vandeven MC (1982) Dynamique de l'amidon en relation avec la croissance et le développement chez le crosne du Japon (Stachys sieboldi, Miq) cultivé à l'obscurité à différentes températures. Thèse de $3^{e}$ cycle. Clermont-Ferrand II 